Results: The interim analysis includes 52 CAPS patients with prior long-term CAN treatment $(43.1 \%$ females) enrolled by September $2018.44 .2 \%$ of the patients participated in the $\beta$-Confident study previously. The mean age was 20.7 years (4.0-79.0 years) at baseline and the mean duration of prior CAN treatment was 5.4 years $(0.0-11.0$ years). 40 patients $(76.9 \%)$ were diagnosed with MWS, the other patients had FCAS (2), NOMID/CINCA (7) or atypical CAPS (1) (subtype diagnosis of 2 patients not available). Mutations in Nod-like receptor family pyrin domain-containing-3 were identified in 34 patients $(68.0 \%)$ including $\mathrm{E} 311 \mathrm{~K}(7)$, Q703K (5), V198M (5), T348M (5), A439V (4). A baseline screening revealed sensorineural hearing loss $(62.0 \%)$, papillitis $(84.0 \%)$ and neurological symptoms $(71.4 \%)$ in patients which had not been detected previously. The majority of patients had no disease related symptoms at baseline and 6 months demonstrating sustained remission in patients receiving long-term CAN treatment. The following disease related symptoms (mild/moderate and severe) were observed in the analysis cohort $(\mathrm{N}=31)$ at baseline and 6 months, respectively - disease symptom: baseline (mild/moderate, severe) vs. 6 months (mild/moderate, severe) - urticarial skin rash: $19.4 \%, 6.5 \%$ vs. $25.8 \%, 0.0 \%$, arthralgia: $32.3 \%, 0.0 \%$ vs. $29.0 \%, 9.7 \%$, myalgia: $9.7 \%, 0.0 \%$ vs. $16.1 \%, 0.0 \%$, headaches: $22.6 \%, 9.7 \%$ vs. $19.4 \%, 19.4 \%$, conjunctivitis: $32.3 \%, 3.2 \%$ vs. $12.9 \%, 6.5 \%$, abdominal pain: $9.7 \%, 6.5 \%$ vs. $22.6 \%, 9.7 \%$. Patients' assessment of disease activity and fatigue did not change between baseline and 6 months. However, at baseline $45.5 \%$ and after 6 months $76.0 \%$ of patients had no impairment of social life by the disease. Serious adverse events were reported for 2 patients (tonsillitis, delivery at week 31).

Conclusion: The RELIANCE study longitudinally monitors the stability of effectiveness of CAN in patients with monogenic periodic fever syndromes. An initial interim analysis including the CAPS subgroup which had prior CAN treatment showed that CAN is an effective and safe treatment in those patients.

Disclosure of Interests: : J. B. Kuemmerle-Deschner Grant/research support from: Jasmin Kuemmerle-Deschner is an employee of University of Tuebingen, Germany, and received consultants/speakers fees from Novartis and SOBI pharmaceuticals and grant support from SOBI and Novartis, Consultant for: Jasmin Kuemmerle-Deschner is an employee of University of Tuebingen, Germany, and received consultants/speakers fees from Novartis and SOBI pharmaceuticals and grant support from SOBI and Novartis, Speakers bureau: Jasmin KuemmerleDeschner is an employee of University of Tuebingen, Germany, and received consultants/speakers fees from Novartis and SOBI pharmaceuticals and grant support from SOBI and Novartis, Norbert Blank Grant/research support from: SOBI and Novartis, Speakers bureau: Novartis and SOBI, Michael Borte Grant/research support from: Pfizer and Shire, Ivan Foeldvari Consultant for: Chugai, Novartis, Gerd Horneff: None declared, Prasad Oommen: None declared, Catharina Schuetz: None declared, Frank Weller-Heinemann: None declared, Julia WeberArden Employee of: Novartis, Tilmann Kallinich Grant/research support from: Novartis, Speakers bureau: Sobi, Roche, Novartis, CLB

DOI: 10.1136/annrheumdis-2019-eular.6224

\section{OP0164 LEFLUNOMIDE IN COMBINATION WITH GLUCOCORTICOIDS IS SUPERIOR TO CONVENTIONAL GLUCOCORTICOIDS MONOTHERAPY IN PREVENTING RELAPSE IN IGG4-RELATED DISEASE: A RANDOMIZED, OPEN-LABEL, CONTROLLED TRIAL}

Yiwen Wang, Zheng Zhao, Jian Zhu, Jianglin Zhang, Feng Huang. Chinese PLA General Hospital, Rheumatology, Beijing, China

Background: Glucocorticoids (GCs) was recommended as first-line therapy for the induction of remission of IgG4-related disease (IgG4-RD), whereas, a large proportion of patients may fail to maintain remission with GCs monotherapy. Considering that recurrent flares might lead to irreversible organ damage, immunosuppressive agents should be added. However, opinions among the experts were split on this issue [1]. Previously, we preliminarily reported the efficacy and safety of GCs and leflunomide (LEF) combination therapy for maintaining the remission of IgG4-RD [2]

Objectives: To evaluate the efficacy and safety of GCs and LEF combination therapy compared with GCs monotherapy for the management of IgG4-RD.
Methods: This study was a 12-month, randomized, open-label, controlled trial (NCT02703194). Patients were randomly allocated to GCs and LEF combination therapy or GCs monotherapy with 1:1 group allocation ratio. The dosage of LEF was $20 \mathrm{mg} / \mathrm{d}$. All received GCs with a predefined taper regimen from a starting dosage of $0.5-0.8 \mathrm{mg} / \mathrm{kg} / \mathrm{d}$. The primary outcome was time to relapse and the main secondary outcomes were time to complete response $(\mathrm{CR})$ and time to remission. Results: A total of 66 patients were randomly assigned (33 in each group) (Figure 1). The demographic and disease characteristics of the patients in the ITT population were similar between the treatment groups. The mean initial dosages of GCs were similar (GCs and LEF: $0.68 \mathrm{mg} / \mathrm{kg} / \mathrm{d}$, GCs: $0.70 \mathrm{mg} / \mathrm{kg} / \mathrm{d}, P=0.529$ ). The cumulative relapse rates at 12 months were $21.2 \%$ in the GCs and LEF group and $50.0 \%$ in the GCs group ( $P=0.023)$. GCs and LEF combination therapy was signif icantly superior to GCs monotherapy with respect to the time to relapse (HR, 0.35; $95 \% \mathrm{Cl}, 0.13$ to $0.90 ; \mathrm{P}=0.030$ ) (Figure 2), and was superior to GCs monotherapy with respect to the time to remission ( $\mathrm{HR}, 1.7495 \% \mathrm{Cl}, 0.97$ to $3.10 ; \mathrm{P}=0.063$ ). These two groups were similar when analyzing the time to $\mathrm{CR}(\mathrm{HR}, 1.5795 \% \mathrm{Cl}$ 0.91 to $2.74 ; \mathrm{P}=0.108)$. At 12 months, $14(42.4 \%)$ patients in the $\mathrm{GCs}$ group and $22(66.7 \%)$ patients in the GCs and LEF group successfully tapered GCs to 10 $\mathrm{mg} / \mathrm{d}$ or less with no relapse $(\mathrm{P}=0.048), 6(18.2 \%)$ patients in the GCs group and $18(54.5 \%)$ patients in the GCs and LEF group to $5 \mathrm{mg} / \mathrm{d}$ or less with no relapse $(P=0.002)$. The relapsing organs of these relapsing patients were salivary glands $(n=5)$, pancreas $(n=5)$, bile ducts $(n=5)$, retroperitoneal fibrosis $(n=3)$, lacrimal gland $(n=1)$, and skin $(n=1)$. The incidences of new-onset diabetes mellitus (GCs and LEF: $21.21 \%$, GCs: $27.27 \%, P=0.566$ ) and the infections (GCs and LEF: $18.18 \%$, GCs: $12.12 \%, P=0.566$ ) were similar between two treatment groups. Conclusion: The efficacy of LEF in combination with GCs therapy was significantly superior to conventional GCs monotherapy in preventing relapse in patients with IgG4-RD. LEF can be used as a steroid-sparing agent in the management of IgG4-RD.

\section{REFERENCES}

[1] Khosroshahi A, Wallace ZS, Crowe JL, et al. International Consensus Guidance Statement on the Management and Treatment of IgG4-Related Disease. Arthritis Rheumatol. 2015 67: 1688-99.

[2] Wang Y, Li K, Gao D, et al. Combination therapy of leflunomide and glucocorticoids for the maintenance of remission in patients with IgG4-related disease: a retrospective study and literature review. Intern Med J 2017; 47: 680-9.

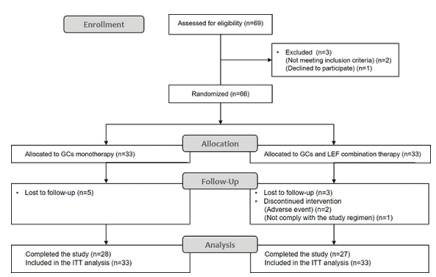

Figure 1. Treatment Assignments and Withdrawal in the Intention-to-Treat Population.

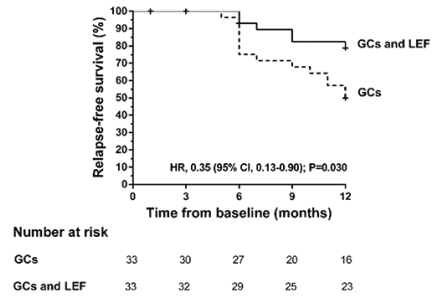

Figure 2. Kaplan-Meier Analysis for Time to Relapse.

Disclosure of Interests: None declared DOI: 10.1136/annrheumdis-2019-eular.5717 